\title{
Another Note on Zeno's Arrow
}

Ofra Magidor

\begin{abstract}
:
In Physics VI.9 Aristotle addresses Zeno's four paradoxes of motion and amongst them the arrow paradox. In his brief remarks on the paradox, Aristotle suggests what he takes to be a solution to the paradox.
\end{abstract}

In two famous papers, both called 'A note on Zeno's arrow', Gregory Vlastos and Jonathan Lear each suggest an interpretation of Aristotle's proposed solution to the arrow paradox. ${ }^{1}$ In this paper, I argue that these two interpretations are unsatisfactory, and suggest an alternative interpretation. In particular, I claim that what seems on the face of it to be Aristotle's solution to the paradox raises two puzzles to which my interpretation, as opposed to Lear’s and Vlastos’s, provides an adequate response.

Key words: Aristotle, Zeno, arrow, paradox, motion

Word Count: 5,261

\footnotetext{
${ }^{1}$ Vlastos (1975), Lear (1981).
} 


\section{\$1 An initial interpretation and two puzzles}

\section{\$1.1 Stating the paradox}

Aristotle’s introduction of the arrow paradox is brief, and rather obscure: ${ }^{2}$

Zeno's reasoning, however, is fallacious, when he says that if everything when it occupies an equal space is at rest, and if that which is in locomotion is always in a now, the flying arrow is therefore motionless.

It is hard to understand what exactly the paradox says, but I shall take the following to be a relatively uncontroversial construal of it: ${ }^{3}$

\section{Arrow-0}

Let $I$ be an interval of time, in which a flying arrow is in motion.

(1) Everything is at rest when it occupies a space equal to itself. (premise)

(2) At every instant $t$ contained in $I$, the arrow occupies a space equal to itself (implicit premise)

(3) At every instant $t$ contained in $I$, the arrow is at rest at $t$. (From (1) and (2))

\footnotetext{
${ }^{2}$ Physics VI.9, 239b5-10 (All translations are by Hardie and Gaye, and taken from Barnes (1995)).

${ }^{3}$ For a defence of a similar construal, see Barnes (1975), pp. 276-283. (The main difference between Barnes's construal and mine is that he takes premise (4) to play a role in establishing (3), rather than in motivating the move from (3) to (5). This difference is unimportant for the rest of my argument). At least one way in which my construal is controversial, however, is that I interpret 'nup' ('now' in the translation used) as 'instant'. For support of this interpretation see Barnes (1975), p. 278 and Pickering (1978). Other alternatives include ‘present instant’ (Lear (1981)), or ‘time atom’ (Valstos (1975), p. 187).
} 
(4) The arrow is always 'at an instant' (premise)

Therefore,

(5) The arrow is motionless in I. (From (3) and (4))

Since from the assumption that the arrow is motion in $I$ it follows, according to the paradox, that the arrow is motionless in $I$ we can conclude that the assumption is wrong and the arrow cannot be in motion in $I$.

Now whatever is meant by the obscure phrase 'occupies a space equal to itself', it seems that the crucial step of the argument as far as Aristotle is concerned is the move from (3) to (5), that is, he is in essence concerned with the following argument: ${ }^{4}$

\section{Arrow-1}

(6) At every instant $t$ contained in $I$, the arrow is at rest at $t$.

Therefore:

(7) The arrow is motionless in $I$.

\section{\$1.2 Aristotle’s solution}

Now here are the two comments Aristotle makes in response to the paradox:

This is false; for time is not composed of indivisible nows any more than any other magnitude is composed of indivisibles. ${ }^{5}$

And:

\footnotetext{
${ }^{4}$ This is endorsed for example by Barnes (1975), p. 282, Owen (1958), p. 216, and Lear (1981), p. 92. ${ }^{5} 239^{\mathrm{b}} 8-10$.
} 
The third is that already given above, to the effect that the flying arrow is at rest, which result follows from the assumption that time is composed of moments: if this assumption is not granted, the conclusion will not follow. ${ }^{6}$

According to the most plausible interpretation of this text, Aristotle is proposing the following: Zeno's argument, as it stands, is invalid (that is, the conclusion (7) does not follow from the premise (6)). Zeno's argument would have been valid, were we to add the additional premise 'time is composed of instants'. ${ }^{7}$ That is, the following argument is valid:

\section{Arrow-2}

(8) At every instant $t$ contained in $I$, the arrow is at rest at $t$.

(9) Time is composed of instants.

Therefore,

(10) The arrow is motionless in $I$.

But Aristotle famously holds the view that (9) is false: lines are not composed of geometrical points, time is not composed of instants, and motion is not composed from motions at an instant. ${ }^{8}$ Since (9) is false, Arrow-2 is unsound and Zeno's conclusion does not follow.

\footnotetext{
${ }^{6} 239^{\mathrm{b}} 30-32$.

${ }^{7}$ Aristotle never literally says this, but it seems strongly conversationally implied by his claim that without this premise the argument is not valid.

${ }^{8}$ See especially Physics VI.1 and On Generation and Corruption I.2.
} 
Barnes complains that there is no reason to attribute (9) to Zeno, and thus Aristotle is unjustly accusing Zeno of presenting an unsound argument. ${ }^{9}$ But this seems to me to misrepresent somewhat the structure of Aristotle's argument. Aristotle is not claiming that Arrow-2 is Zeno's argument. Rather, he is claiming that Zeno is making the invalid Arrow-1. As Aristotle sees it, the reason why Zeno seems to think it is a valid argument is because Zeno is implicitly assuming (9), or at least this is the only way to explain why Zeno's argument seems plausible. ${ }^{10}$ But Zeno cannot amend his argument by including (9) explicitly, for then he would end up with the unsound Arrow-2. So Zeno’s argument fails.

This initial construal of Aristotle’s solution raises two puzzles:

(Puzzle-1) Aristotle's claim that Arrow-1 is invalid as it stands seems quite plausible. If time intervals are not composed of instants, there is no reason to think that any properties of the instants should be properties of the intervals. If I am not composed of metal, there is no reason to think that if metal is a good conductor, then so am I.

What is not clear is why we should think that the amended Arrow-2 is valid. As Barnes notes, by suggesting that Arrow-2 is valid, Aristotle seems to commit an instance of 'the fallacy of composition': the claim that if $\mathrm{O}$ is composed Fs, then if each of the Fs has a property $\mathrm{P}$, then $\mathrm{O}$ has the property $\mathrm{P} .{ }^{11}$ This claim is, of course, false. For example, it could be that I am composed of atoms, all my atoms are smaller

\footnotetext{
${ }^{9}$ Barnes (1975), p. 282.

${ }^{10}$ A similar point is made by Vlastos (1975), p. 189.

${ }^{11}$ Barnes (1975), p. 283.
} 
than a pinhead, and yet I am not smaller than a pinhead. Ideally, we would like to have an interpretation that does not attribute such a blatant fallacy to Aristotle.

(Puzzle-2) As we shall see in the following sections, most commentators (including myself) believe that Aristotle rejected premise (8), namely the claim that at every instant $t$ contained in $I$, the arrow is at rest at $t$. Given this, it is highly surprising that Aristotle does not (at least not explicitly) attack Zeno’s argument on the grounds of its being based on a false premise: Zeno's argument is attacked on the grounds of being invalid, not on the grounds of being unsound.

I thus take it that it is desirable to find an interpretation of Aristotle's solution to Zeno's arrow paradox that provides an adequate response to puzzle-1 and puzzle-2, and in §4 I will suggest such an interpretation.

\section{§2 Being in motion and at rest at an instant}

Before I turn to discuss the various interpretations of Aristotle's solution to the paradox, there is one important issue that requires further elaboration. In stating puzzle-2 above, I have mentioned that one point of agreement between the interpretations I reject and my own interpretation is that we all maintain that Aristotle rejected premise (8), that is, we all maintain that Aristotle rejected the claim that the arrow is at rest at every instant contained in $I$. However, as will become apparent, our reasons for attributing this view to Aristotle are importantly different, and rely on a different understanding of Aristotle's position on the general question of whether it is in principle possible for an object to be in motion or at rest at an instant. Let me therefore briefly discuss the issue of Aristotle’s position on this general question. 
It is a widely accepted orthodoxy that Aristotle held the view that nothing can be in motion or at rest at an instant. ${ }^{12}$ This is usually based on the thought that saying ' $\mathrm{O}$ is in motion (rest) at an instant $t^{\prime}$ involves some sort of category mistake, that is, the concepts 'being in motion at $x$ ' or 'being at rest at $x$ ' are in some sense applicable only to time intervals, not to instants. ${ }^{13}$

The fact that this claim is so widely accepted is hardly surprising. Several passages in Aristotle seem to support it directly, especially the discussion in Physics VI.3 (234 $24-32)$, in which Aristotle explicitly says "nothing can be in motion in a now... Nor can anything be at rest”. ${ }^{14}$

Nevertheless, I think this orthodoxy is wrong. Benjamin Morison has recently argued that this passage from Physics VI.3, namely 'oupee eh t\%anum ki nei bai', should not be translated as saying that nothing can be moving at an instant, but rather as saying that nothing can be moving during an instant. ${ }^{15}$ The idea is that there cannot be any instantaneous motions (sometimes called 'jerk motions') - no motion or rest can be produced in an instant, and no object can be in the state of motion or in the state of

\footnotetext{
${ }^{12}$ See for example Barnes (1975), p. 281, Lear (1981), p. 92, Moore (2001), p. 42, Pickering (1978), p. 256, Ross (1936), p. 71 and Vlastos (1975), p. 192. I am ignoring here the different terminology used by different writers to make this claim, e.g. the use of 'in an instant' rather than 'at an instant'.

${ }^{13}$ This claim is sometimes (e.g. by Barnes (1975)) taken to say that ' $\mathrm{O}$ is in motion at $t$ ' is simply false, sometimes (e.g. by Vlastos (1975)) that it is senseless.

${ }^{14}$ see also $239^{\mathrm{b}} 1-2$.

${ }^{15}$ Morison (MS), pp. 15-16.
} 
rest merely for an instant. ${ }^{16}$ But this does not imply that an object cannot be in the states of motion and rest at an instant at all.

This interpretation can be defended on several grounds. Almost everything that Aristotle says in Physics VI.3 still makes sense under the new interpretation. ${ }^{17}$ In particular, it seems better to fit Aristotle’s conclusion of this chapter (234 $\left.{ }^{\text {b}} 8\right)$ : “It follows that the motion of that which is in motion and the rest of that which is at rest must occupy time”. Aristotle is not saying here that the state of being in motion must occupy time, but that the production of motion or rest must take time. And as Morison argues, this interpretation also seems necessary if we are to make sense of Aristotle's arguments in Physics VI.5. To point to one consideration: in $236^{\mathrm{a}} 18$, Aristotle says "if the changing thing is at rest in the whole time CA..., it is at rest in A also”. This is at least one passage in which Aristotle seems perfectly content to explicitly attribute the property of being at rest at an instant to an object.

Another argument against the thesis that Aristotle held nothing can be in motion or at an instant is the following: if this claim were true, then it is hard to see how we can provide an adequate interpretation of Aristotle’s solution to Zeno’s arrow paradox, and in particular one which addresses puzzle-2: if nothing can be in motion or at rest at an instant, claim (6) would be obviously false, and Aristotle could have easily dismissed the paradox on the grounds of being unsound.

\footnotetext{
${ }^{16}$ The latter claim with respect to rest is problematic when we consider the extreme positions of a swinging pendulum or a bouncing ball (see Morison (MS), n. 10 and Bostock (1973)).

${ }^{17}$ An exception might be Aristotle's third argument in $234^{\mathrm{a}} 34-234^{\mathrm{b}} 4$. I admit that this argument poses a serious challenge to the interpretation I am adopting.
} 
So much for what Aristotle does not claim. But what does Aristotle think about being in motion or rest at an instant? An illuminating discussion appears in Physics VI.10. Aristotle discusses here the question of whether a geometrical point can move in isolation. He argues that it is impossible for a point to move in isolation, but only "in so far as the body or the magnitude to which it belongs is in motion, just as that which is in a boat may be in motion in virtue of the motion of a whole”. ${ }^{18}$

An equivalent principle, I think, should apply to motion at an instant. There cannot be independent motion at an instant. However, it can still be true that at an instant a body is in motion, in so far as that instant belongs to an interval of time in which there is motion. The upshot is that it is not a category mistake to say that a body is in motion or at rest at an instant. What is true is that if a body is in motion (rest) at an instant, this fact is derivative on its being in motion (rest) at a stretch of time containing this instant.

It is important to understand that this is not a semantic claim. ${ }^{19}$ I have not said that Aristotle defines a new sense of the phrase 'being in motion'. He has not stipulated that ' $\mathrm{O}$ is in motion at $t$ ' means the same as ' $\mathrm{O}$ is in motion at $I$, and $t$ is contained in I'. Rather, this is a metaphysical claim. If anything is in motion at an instant, this must be ontologically dependent on the fact that it is motion in a stretch $I$ that contains $t .^{20}$

\footnotetext{
${ }^{18} 240^{\mathrm{b}} 8-11$.

${ }^{19}$ Contra Owen (1958), p. 220.

${ }^{20}$ Cf. Aristotle’s notion of being 'prior by nature' (e.g. in Categories 12 or Posterior Analytics I.2).
} 
It is quite clear that Aristotle thinks that geometrical points exist, though only derivatively on magnitudes. He thinks that time instants exists, though only derivatively on time intervals. Given his general view that time, motion, and magnitude have isomorphic structures, ${ }^{21}$ it is only natural to suppose that Aristotle equally held that 'motion instants' exist - though only derivatively on 'motion intervals'.

\section{§3 Two interpretations rejected}

I now turn to discuss Lear and Vlastos's interpretations to Aristotle's solution. Both interpretations share the assumption that according to Aristotle nothing can be in motion or at rest at an instant. As I have explained above, I think this assumption is wrong and this is one objection I have to both these interpretations. In what follows, however, I provide some independent reasons for rejecting these interpretations.

\section{§3.1 Vlastos’s interpretation}

The first interpretation I want to discuss is that of Vlastos. ${ }^{22}$ Vlastos claims that, contrary to my construal of Aristotle's solution, Aristotle thinks the move from (6) to (7) (that is, Arrow-1) is valid. But Aristotle is clearly attacking the validity of some argument. According to Vlastos, what Aristotle is attacking is the validity of the move from:

(11) For every instant $t$ contained in $I$, the arrow is not in motion at $t$.

to

(12) For every instant $t$ contained in $I$, the arrow is at rest at $t$.

\footnotetext{
${ }^{21}$ See for example Physics IV.11 219 11 , Physics VI.1 231 ${ }^{\mathrm{b}} 19$, and Physics VI.4 $235^{\mathrm{a}} 14$.

${ }^{22}$ Vlastos (1975). This is a reconstruction of Vlastos’s claims using my own terminology.
} 
The reason, according to Vlastos, is that Aristotle thinks that there cannot be motion and rest at an instant (presumably, the claim that time is not composed of instants is supposed to remind us of this point). It follows that while (11) is true (because the arrow cannot be in motion at $t$ ), ${ }^{23}$ (12) is not true. So (12) (which is identical with premise (6) in Arrow-1) should not be accepted, and therefore Zeno's argument is unsound. In other words, Vlastos assumes that the only reason to suppose (12) is true in the first place is because it is the conclusion of an implicit 'pre-argument' - the one from (11) to (12). But because the pre-argument is invalid, we should not take (12) to be true.

In a sense Vlastos's interpretation offers a response to both puzzle-1 and puzzle-2, but only at the price of making his interpretation implausible. The response to puzzle-1 is that Arrow-1 is already valid, and hence Arrow-2 is trivially valid (without any special appeal to the premise concerning composition). And the response to puzzle-2 is that according to Vlastos, Aristotle is attacking the soundness of the argument, although he does so only implicitly, via his attack on the validity of the pre-argument that is supposed to supply the grounds for accepting premise (6).

But this response to puzzle- 1 is unsatisfying because Vlastos offers no explanation for why we should take Arrow-1 to be valid. And the response to puzzle-2 is unsatisfying because it does not bear much resemblance to Aristotle's discussion of the paradox as it appears in the text. Aristotle presents the paradox as resting on the claim that the

\footnotetext{
${ }^{23}$ Note, however, that this is already at tension with Vlastos's claim that "To say that the arrow is moving in any instant would be (strictly speaking) senseless” (p. 192). One would expect that negating a senseless sentence would result in a senseless sentence, rather than in a true one.
} 
arrow is at rest (rather than that it is not in motion) at every instant. One would expect that if the main point of Aristotle's solution lies in rejecting the move from (11) to (12) we would see some mention of that in the text. ${ }^{24}$ Moreover, recall that Aristotle accuses Zeno of committing a fallacy when he claims that if 'everything when it occupies an equal space is at rest' and 'that which is in locomotion is always in a now' then the flying arrow is motionless. It is hard to see how Aristotle's attack on the acceptability of this conditional can be correctly interpreted as an attack on its antecedent.

Another problem with Vlastos's interpretation is this. We can restate Zeno's paradox in the following terms: ${ }^{25}$

\section{Arrow-3}

(13) For every instant $t$ contained in $I$, the arrow is not in motion at $t$.

Therefore,

(14) The arrow is motionless in $I$.

This new paradox seems just as devastating as Zeno's original paradox, and now Vlastos cannot claim that it is unsound. Vlastos seems to anticipate this objection, and claims that the new argument would be invalid (in a way that the original Arrow-1 was not). ${ }^{26}$ His reasoning is dense and hard to follow, but as far as I understand it

\footnotetext{
${ }^{24}$ Admittedly, Aristotle's preceding remarks at the end of Physics VI.8 can be plausibly read as making this point, but it is striking that Aristotle does not seem to allude to these remarks in his discussion of the paradox in Physics VI.9.

${ }^{25}$ This objection is raised in Barnes (1975), p. 279.

${ }^{26}$ Vlastos (1975), pp. 191-192.
} 
goes along the following lines: (13) is only true because it is a category mistake to say that the arrow is in motion at $t$, that is, it is only true for what Vlastos calls 'semantic reasons'. Therefore we should not expect that the property of not being in motion will 'pass on' to the conclusion (14). But this response is unconvincing: one can think, for example, that it is for similar 'semantic reasons' that no part of my wall is left-handed (that is, because it is some kind of category-mistake to say that a part of my wall is left-handed), and correctly conclude, that (again, for 'semantic reasons') my whole wall is not left-handed. It is thus far from clear that the mere introduction of 'semantic reasons' which are allegedly present in Arrow-3 and lacking in Arrow-1 is sufficient to justify Vlastos's claim that while Arrow-1 is valid, Arrow-3 is not.

\section{§3.2 Lear's interpretation}

I now turn to discuss Lear's interpretation which is, in my view, more plausible. $^{27}$ Lear claims that Aristotle has two separate solutions to the paradox. Firstly, Aristotle thinks that Arrow-1 is invalid, because the move from (6) to (7) requires the additional premise (9). In addition, he claims, Aristotle thinks that Arrow-1 is unsound, because he thinks that nothing can be in motion or at rest at an instant, and hence premise (6) is false.

Lear's interpretation clearly avoids some of my objection to Vlastos’s interpretation: Lear shares my view that the argument the validity of which is questioned in Aristotle’s passage is Arrow-1 (rather than the pre-argument suggested by Vlastos). And his interpretation clearly avoids my last objection to Vlastos, because on his view Arrow-3 will be invalid, just as Arrow-1 is.

\footnotetext{
${ }^{27}$ Lear (1981).
} 
However, Lear provides us with no answers to the two puzzles I raised. He provides no adequate explanation for why Arrow-2 is valid. Nor does he explain why Aristotle does not attack the falsity of Zeno's premise explicitly. His interpretation seems to make the fallacious move from:

(i) Aristotle holds the view that nothing can be in motion or at rest at an instant.

And

(ii) The claim that nothing can be in motion or at rest at an instant could in principle be used to solve Zeno's paradox, since it entails that Zeno’s argument is unsound.

To:

(iii) Aristotle actually uses the claim the nothing can be in motion or at rest at an instant to solve the paradox.

Even if (i) and (ii) were true, (iii) is not - or at least we have no textual evidence that supports it.

\section{$\S 4$. An alternative interpretation}

I now present my alternative interpretation. Aristotle is, I suggest, envisaging two opposing metaphysical views. According to one (in which time is composed of instants), time instants are ontologically and explanatorily prior to time intervals. According to the other (that endorsed by Aristotle), it is the intervals that are metaphysically prior to the instants.

Zeno, Aristotle is claiming, is implicitly assuming the former picture. He has told us some story about why the arrow is at rest at every instant - let us not argue with this 
story for the time being. But in order to conclude from this that the arrow is motionless in $I$, we must assume that the properties of the instants are relevant for deriving the properties of the interval, and this claim only holds in the metaphysical picture that Zeno is implicitly assuming, and Aristotle rejects.

How does this interpretation help solve my two puzzles?

Recall, that puzzle-1 asked why Arrow-2 was valid, which under my current suggestion amounts to asking why, if we were to adopt Zeno's metaphysical picture, premise (7) would follow from premise (6). Initially, it is not obvious why recasting the question in these terms helps solve puzzle-1: even if we accept that instants are ontologically prior to intervals, there is no reason to think that any property of the instants will be a property of the interval. At best, we can assume that all properties of the interval supervene on properties of the instants. For example, even if I think my atoms are ontologically prior to me, I can still think that all my atoms are small and I am big. My size merely supervenes on the size of my atoms and their arrangement.

The problem can be addressed if we distinguish between the following two claims:

Claim-1: If O is composed of Fs, then for every property $\mathrm{P}$, if each of the Fs has $\mathrm{P}$ then $\mathrm{O}$ has $\mathrm{P}$.

Claim-2: If O is composed of Fs, then for some privileged property P, if each of the Fs has $\mathrm{P}$ then $\mathrm{O}$ has $\mathrm{P}$. 
Claim-1 is a fallacy. Claim-2 is not, provided that we have some reason to think that $\mathrm{P}$ is indeed privileged in the relevant way. For example, I think that the fact that each of my atoms are in a certain location (say the library), does entail that I am currently in that location.

Aristotle, I am suggesting, thinks that being in motion is exactly one of those privileged properties. As he sees it, the most plausible way to adopt the view that motion and rest at an interval can be explained via motion and rest at an instant is to accept that being at motion (rest) at every instant entails being at motion (rest) in the corresponding interval. Versions of the view according to which motion and rest at an interval supervene on motion and rest at instants in other, more complicated ways, are simply taken to be metaphysically implausible. ${ }^{28}$

How about puzzle-2? In §2, I have claimed that Aristotle thinks that if $\mathrm{O}$ is in motion at an interval of time, then it is in motion at each instant contained in that interval. Since Aristotle obviously believes that the arrow is in motion at $I$, then he must believe that it is in motion at every instant of $I$, and therefore that it is not at rest at any (and a fortiori not at every) such instant. So according to my view Aristotle thinks that Zeno’s premise (6) is false.

\footnotetext{
${ }^{28}$ A similar point is made by Aristotle in On Generation and Corruption I.2 (316 $\left.30-34\right)$, regarding why magnitudeless points cannot 'add up' to make an interval with a magnitude. It's important to note that the argument here - whether or not it is successful - is clearly not an instance of the fallacy of composition. For further support of this interpretation see also the principles of non-supervenience in White (1992), pp. 13, 29, and 47.
} 
Why then hasn't Aristotle dismissed Zeno's argument as being unsound? The answer easily emerges from the reasoning I have sketched in the above paragraph: in order for Aristotle to argue that (6) is false, he must start from the premise that the arrow is in motion at $I$. But of course he cannot give this argument in response to Zeno, for that would be completely question begging. Aristotle believes that Zeno's argument is based on a false premise, but cannot argue for that without already claiming that Zeno's conclusion is false. This is why he chooses to attack only the validity of the argument.

Finally, there is one serious objection to my interpretation. ${ }^{29}$ According to the view I presented in section $\S 2$, it seems to come out that $\mathrm{O}$ is in motion (at rest) at a stretch $I$ if and only if it is in motion (at rest) at every instant contained in I. It is quite clear why the 'only if' direction of this claim is supposed to hold: on the picture I have presented the instants inherit the motion properties from the interval in which they are contained. The 'if' direction of this claim allegedly holds because if for each instant $t$ contained in $I$, $\mathrm{O}$ is in motion at $t$, then this must derive from the fact that $\mathrm{O}$ is in motion in $I .^{30}$

But now comes the objection: doesn't it turn out that according to Aristotle's view Arrow-1 is valid after all? If the arrow is at rest at every instant, this must be because

\footnotetext{
${ }^{29}$ This objection is suggested in Owen (1958), p. 220.

${ }^{30}$ Actually, this is not strictly speaking true: it could be that the fact that $\mathrm{O}$ is in motion at $t$ is derivative on O's being in motion at some sub-interval of $I$, leaving open the possibility that $I$ can be 'covered' by sub-intervals in which $\mathrm{O}$ is in motion, although $\mathrm{O}$ is not in motion at $I$. It's quite clear however, that Aristotle would endorse this possibility and so I leave this point aside.
} 
it is at rest for the whole interval, so it is at rest at $I$, that is, Zeno's conclusion follows from premise (6) after all.

This objection is quite ingenious, but it does not work. First, we should note that the above argument does not show that Arrow-1, as it stands, is valid. At best it shows that the following argument is valid:

\section{Arrow-4:}

(15) At every instant $t$ contained in $I$, the arrow is at rest at $t$.

(16) The properties of instants are derived from the properties of intervals (that is, the correct metaphysical view is the one that I have attributed to Aristotle).

Therefore,

(17) The arrow is motionless at $I$.

The point is that Arrow-1, as it stands, is invalid unless we explictly add either the premise that Zeno's metaphysical picture is correct (that is, that time is composed of instants), or the premise that Aristotle's metaphysical picture is correct (that is, that time is not composed of instants, and moreover that the properties of instants derive from the properties of intervals). But these are not the only two options: one could think that there was simply no relation between the properties of instants and the properties of the corresponding intervals and therefore that neither picture is correct, in which case (17) will not follow from (15). Thus without adding any further premises Arow-1 is indeed invalid. 
Second, and importantly, Arrow-4 (which Aristotle would presumably take to be valid) is not a paradox. For if we accept the metaphysical picture suggested by (16), the only way Zeno could defend premise (15) is by assuming that the arrow is at rest at $I$. But now it would be Zeno who is begging the question! All that the validity of Arrow-4 shows is that if the arrow is at rest at $I$ (which is the only case that would make (15) true), then the arrow is at rest at $I$. Hardly a paradox.

To sum up: Aristotle is suggesting that we consider two competing metaphysical pictures. Zeno's argument is initially plausible, because it implicitly assumes a picture where telling us some story about the arrow's independent state at an instant (that is, its 'occupying an equal space') should entail its state at the interval $I$. In the alternative metaphysical view, no such independent property of the arrow at an instant can tell us anything about its state at $I$. Furthermore, since the arrow is in fact moving at $I$, Zeno was wrong to think that the arrow was at rest at every instant. As long as we are willing to accept Aristotle's alternative picture, the paradox is refuted. This result is achieved without directly engaging with the elusive claim that if at an instant the arrow is 'occupying an equal space' then it must be at rest at that instant. ${ }^{31}$

\footnotetext{
${ }^{31}$ Thanks to audiences at Cambridge and at Oxford, as well as to Markus Kohl, Gerald Massey, Ben Morison, and an anonymous referee for their helpful comments on this paper.
} 


\section{References}

Barnes (1975) Barnes, J., The Presocratic Philosophers (vol. 1), Routledge.

Barnes (1995) Barnes, J. (ed.), The Complete Works of Aristotle, Princeton University Press.

Bostock (1973) Bostock, D., 'Aristotle, Zeno, and the Potential Infinite', Proceedings of the Aristotelian Society 73, pp. 37-57.

Lear (1981) Lear, J., ‘A Note on Zeno’s Arrow’, Phronesis 26, pp. 91-104.

Moore (2001) Moore, A.W., The Infinite (2ed edition), Routledge.

Morison (MS) Morison, B., 'Aristotle on Primary Time in Physics VI' (unpublished manuscript).

Owen (1958) Owen, G.E.L., 'Zeno and the Mathematicians', Proceedings of the Aristotelian Society 58, pp. 199-222.

Pickering (1978) Pickering, F.R., 'Aristotle on Zeno and the Now', Phronesis 23 (1978), pp. 253-257.

Ross (1936) Ross, W.D., Aristotle’s Physics, Clarendon Press.

Vlastos (1975) Vlastos, G., ‘A Note on Zeno’s Arrow’, in Furley, D. J. and Allen, R. E. (eds.), Studies in Presocratic Philosophy (vol. 2), Routledge.

White (1992) White, M. J., The Continuous and the Discrete, Clarendon Press. 